\title{
Observation of two separate bipolar spindles in the human zygote
}

\author{
Xiaoming $\mathrm{Xu}^{1} \cdot$ Linheng $\mathrm{Li}^{2,3} \cdot$ Chuilian Zhang ${ }^{4} \cdot$ Li Meng $^{4,5}$ (D) \\ Received: 9 January 2019 / Accepted: 13 March 2019 / Published online: 30 March 2019 \\ (C) Springer Science+Business Media, LLC, part of Springer Nature 2019
}

\section{Dear Editor-in-Chief:}

The article by Reichmann and colleagues recently published in Science [1] raises a number of issues regarding the earliest stages of mammalian embryo development. They report the existence of two distinct and spatially separate bipolar spindles in mouse zygotes, which appear to function independently during the first mitotic division. These observations beg the question of whether human zygotes may exhibit a similar spindle assembly and function mechanism. Here, we report a similar situation where a human zygote containing two individual spindles was observed in the course of our ongoing research. As shown in Fig. 1, the zygote in question was fixed in anaphase of the first cell cycle and processed for confocal fluorescence microscopy after labeling the sample with DAPI (for chromatin blue), NuMa (red), and tubulin (for spindle, green following standard immunofluorescent protocols). What is apparent is that the long axis of the two spindles is aligned in parallel with each other with a radiating array of microtubules emanating from both spindle poles. That a solitary microtubule organizing center is evident at one pole (upper) is interesting given the role of the paternal centrosome in human embryos. At anaphase, the spindles' long axes align the two separated groups of chromosomes (A) between their two poles.

Given that this letter documents an isolated case of dual spindles in a human zygote, more work is needed to establish whether this is a common feature of cell cycle M-phase or a

Li Meng

Limengivf@gmail.com

1 Beijing Perfect Family Hospital, Xicheng District, Beijing, China

2 Stowers Institute for Medical Research, Kansas City, MO, USA

3 Department of Pathology and Laboratory Medicine, University of Kansas City, Kansas, MO, USA

4 Reproductive Medicine Center, Henan Provincial People's Hospital, Zhengzhou, Henan, China

5 LA IVF Clinic, Los Angeles, CA, USA fortuitous finding that could represent abnormal processing of the maternal and paternal genomes. Moreover, it will be necessary to couple this approach with one that tracks gender-specific methylation as reported by Mio and colleagues [2]. The potential advantage of dual-spindle formation at the zygote stage of development hinges upon the fact that the spindles segregate two very different sets of chromatin: sperm DNA is highly compact; its genome relatively methylated and its transcription inert. Chromatin in ovulated oocytes, however, is less compact, and its genome is relatively undermethylated. If this finding demonstrates some degree of prevalence in multiple embryos, then it suggests that upon fertilization, spatial separation of parental chromosomes as observed effects compartmentalization for chromatin reprogramming that in a gender-specific way would include protamine-histone replacement, epigenetic modifications, and chromatin remodeling. In mice, this separation of localized chromatin modifications is conserved until the four-cell embryo stage and then gradually disappears [3]. We do not know how long separation of parental genomes continues during human preimplantation development findings consistent with segregation of genomes have been reported by Kai et al. in 0PN human zygotes [2]. Dual-spindle formation in human zygotes may be extremely transient or possibly coincides with the so-called $0 \mathrm{PN}$ embryo. The failure of pronuclei to migrate to the cell center and align the two spindles, and asynchronous parental nuclear envelope breakdown (NEBD), both increase the possibility of heterogonic division that has been proposed to underlie the propagation of gender-specific spindle assembly, multinucleation, mosaicism, and arrested development [4]. This would be consistent with conditions such as mosaicism, chimerism, and mixoploidy, which frequently are observed after preimplantation genetic testing in the clinical practice of IVF. If confirmed, this finding raises the specter of a parental genome separation during the first cell cycle in the human embryo that would require rethinking our current concepts of syngamy [5]. This, in turn, could raise questions about when human life begins [6]. 
Fig. 1 Immunofluorescence staining of human zygote fixed at anaphase of the first mitosis. a Maternal and paternal chromosomes at anaphase are spatially separated. b Two distinct spindles separated were observed and parallel to each other. $\alpha$ Tubulin (green), NuMA (red), and chromosomes (blue). Bar $=$ $30 \mu \mathrm{m}$ b $\alpha-$ tubulin/NuMA/DAPI
Acknowledgements We thank Alyssa Meng (University of California At Santa Barbara) for editing of the manuscript.

\section{References}

1. Reichmann J, Nijmeijer B, Hossain MJ, Eguren M, Schneider I, Politi AZ, et al. Dual-spindle formation in zygotes keeps parental genomes apart in early mammalian embryos. Science (New York, NY). 2018;361:189-93.

2. Kai Y, Moriwaki H, Yumoto K, Iwata K, Mio Y. Assessment of developmental potential of human single pronucleated zygotes derived from conventional in vitro fertilization. J Assist Reprod Genet. 2018;35:1377-84.

3. Mayer W, Smith A, Fundele R, Haaf T. Spatial separation of parental genomes in preimplantation mouse embryos. J Cell Biol. 2000;148: $629-34$.
4. Destouni A, Vermeesch JR (2017) How can zygotes segregate entire parental genomes into distinct blastomeres? The zygote metaphase revisited. Bioessays 39(4). https://doi.org/10.1002/bies.201600226

5. Albertini DF. Making sense out of syngamy at the onset of mammalian development. J Assist Reprod Genet. 2018;35:1357-8.

6. Paulson RJ. The unscientific nature of the concept that "human life begins at fertilization," and why it matters. Fertil Steril. 2017;107: 566-7.

Publisher's note Springer Nature remains neutral with regard to jurisdictional claims in published maps and institutional affiliations. 